\title{
High-pressure operation of a xenon-GPSC/MSGC hybrid detector for hard X-ray spectrometry
}

\author{
L.C.C. Coelho ${ }^{\text {a }}$, J.F.C.A. Veloso ${ }^{a}$, , $*$, D.S. Covita ${ }^{a}$, L.F. Requicha Ferreira ${ }^{a}$, \\ J.M.F. dos Santos ${ }^{\mathrm{a}}$ \\ ${ }^{a}$ Departamento de Fisica da Universidade de Coimbra, 3004-516 Coimbra, Portugal \\ ${ }^{\mathrm{b}}$ Departamento de Física da Universidade de Aveiro, 3810-193 Aveiro, Portugal
}

Received 19 February 2006; received in revised form 1 August 2006; accepted 11 September 2006

Available online 11 October 2006

\begin{abstract}
The performance of a high-pressure xenon gas proportional scintillation counter/microstrip gas chamber (GPSC/MSGC) hybrid detector has been investigated for filling pressures from 1 up to $10 \mathrm{bar}$, for 22-, 30- and 60-keV photons. GPSC/MSGC hybrid detectors are based on a xenon-GPSC instrumented with a CsI-coated microstrip plate photosensor placed directly within the xenon envelope, as a substitute for the photomultiplier tube. This design avoids the constraints due to the use of a quartz scintillation window for GPSC-photosensor coupling, which absorbs a significant amount of scintillation and is a drawback for applications where large detection areas and high filling pressures are needed. The lowest energy resolutions are achieved for 2 bar $(5.5 \%$ and $3.4 \%$, FWHM, for 22- and $60-\mathrm{keV}$ photons, respectively). Increasing the pressure to the 5-6 bar range, competitive energy resolutions of $7 \%$ and $4.5 \%$ are still achieved for 22- and 60-keV photons, respectively. This detector could be a compelling alternative in applications where compactness, large detection area, insensitivity to strong magnetic fields, room temperature operation, large signal-to-noise ratio and good energy resolution are important requirements.
\end{abstract}

(C) 2006 Elsevier B.V. All rights reserved.

PACS: $07.85 . \mathrm{Nc} ; 29.40 . \mathrm{Cs} ; 29.40 . \mathrm{Mc} ; 85.60 . \mathrm{Ha}$

Keywords: Gas scintillation; High pressure; MSGC; Csl photocathode; X-rays; Gamma-rays

\section{Introduction}

High-pressure xenon gas detectors with large area detection capability are attractive for hard X-ray detection applications such as X-ray digital radiography, synchrotron radiation studies, crystallography, $\mathrm{X}$ - and gamma-ray astronomy. In the last decade, micropattern electron multiplier systems, such as MSGC [1] and GEM [2], which are faster and present a much higher count rate capability, substituted for the often-used MWPC technology. Nevertheless, gaseous detectors based on electron avalanche amplification present a strong reduction in the charge gain as the gas pressure increases. The single and double GEM

\footnotetext{
*Corresponding author. Departamento de Física da Universidade de Aveiro, 3810-193 Aveiro, Portugal.

E-mail address: jveloso@fis.ua.pt (J.F.C.A. Veloso).
}

electron multipliers present a reduction of about a factor of 30 when the pressure increases from 1 to 3 bar [3]; the MSGC gain drops by about a factor of 15 when the pressure increases from 1 to 5 bar [4], while for the GEM + MSGC and the triple GEM multipliers, the gain drops by about two and four orders of magnitude, respectively [4,5], for the same pressure increase.

On the other hand, gas proportional scintillation counters (GPSCs) [6] are based on the production of secondary scintillation in the gas as the signal amplification, and present improved energy resolution, high signalto-noise ratio and high counting rates with reduced spacecharge effects, when compared to gaseous detectors based on electron avalanche amplification. Photomultiplier tubes (PMTs) have been the photosensors of choice for the readout of the VUV scintillation light produced in the noble gas fillings of GPSCs. However, the use of a PMT 
limits the detection area and, for high-pressure gas fillings, thick quartz windows are needed for the GPSC-photosensor coupling. These absorb a significant amount of scintillation and are a drawback for applications where large detection areas are needed.

In response to these limitations, we developed the gas proportional scintillation counter/microstrip gas chamber (GPSC/MSGC) hybrid detectors $[7,8]$. These detectors are based on a xenon-GPSC instrumented with a CsI-coated microstrip plate (MSP) photosensor placed directly within the xenon envelope as a substitute for the PMT, avoiding, this way, the PMT area limitations and the quartz scintillation window constraint. The CsI photocatode was chosen for its high quantum efficiency in the VUV region and for its simple fabrication [9]. The feasibility of detecting vacuum ultraviolet (VUV) photons using an MSGC with a thin layer of CsI deposited on the MSP as the photocathode, operating in reflective mode, was demonstrated by Zeitelhack et al. [10,11]. The presence of the CsI-coating does not compromise the operation of the MSP. On the other hand, it results in a reduced substrate charge build-up, since its semiconductive characteristics are comparable to those of the semiconducting glass substrates, namely a volume resistivity of $10^{11}-10^{12} \Omega \mathrm{cm}$ [12], used for high-rate MSP applications, e.g. Ref. [13].

Although the energy resolution achieved with a xenonGPSC/MSGC hybrid detector (11\% FWHM at $6 \mathrm{keV}$ [8]) is not as good as that of PMT-based GPSCs ( $8 \%$ FWHM at $6 \mathrm{keV})$, the performance is better than other xenon detectors based on charge amplification. This detector could be a compelling alternative in applications where compactness, large detection area, insensitivity to strong magnetic fields, room temperature operation, large signalto-noise ratio and good energy resolution are important requirements $[14,15]$.

The detector performance is limited by optical positive feedback resulting from the additional scintillation light produced in the charge avalanche at the MSP anodes, in the absence of quenching, limiting the voltage difference applied between cathode and anode strips of the MSP. The lowest energy resolution is achieved at the onset of the positive feedback effect, which introduces additional statistical fluctuations that lead to a degradation of the detector energy resolution. On the other hand, the use of reduced gains at the optimum operating conditions of the MSGC results in a negligible charge build-up and prevents the possibility of MSP discharge breakdown.

Former results for xenon pressure fillings, ranging from atmospheric pressure up to 2.8 bar [16], have shown a fast decrease in the detector pulse amplitude, of almost $40 \%$, when the pressure increases from 1 to 2 bar, and a much slower decrease for higher pressures, without a degradation of the detector energy resolution for high-energy X-rays. In this work, we investigate the performance of a highpressure GPSC/MSGC hybrid detector: detector energy resolution, total gain and light gain as a function of gas pressure, from 1 to $10 \mathrm{bar}$, and for X-ray energies in the range of $22-60 \mathrm{keV}$. High pressure gas fillings extend the detection range to hard X-rays and/or allow a more compact detector design.

\section{Detector description and experimental setup}

The schematic of the GPSC/MSGC hybrid detector used in this work and its operation principles are shown in Fig. 1. The radiation window and the focusing electrode, $F$, are maintained at a negative high-voltage, $-\mathrm{HV}_{0}$, while the mesh G1 $(80-\mu \mathrm{m}$ diameter stainless steel wire with $900-\mu \mathrm{m}$ spacing) and its holder are kept at - HV1; the voltage difference defines the electric field in the $3-\mathrm{cm}$ thick absorption region, which is kept below the xenon scintillation threshold. A Macor piece, glued with low vapour pressure epoxy to the detector body and to the radiation window holder, is used for electrical insulation of the different electrodes. The holder of G1 is fixed to the Macor with screws, one of which is used as a feedthrough for the G1 biasing voltage. The mesh G1 and the MSP limit the $0.4-\mathrm{cm}$ thick scintillation region. The MSP backplane and cathode strips are maintained at ground potential, while a positive voltage, $V_{\mathrm{a}}$, is applied to the anode strips.

Primary electron clouds from X-ray interactions in the absorption region drift, under the influence of a weak electric field, towards the scintillation region. The electric field in the scintillation region is high enough for each primary electron to gain enough energy between collisions to excite but not ionize the xenon atoms, producing VUV

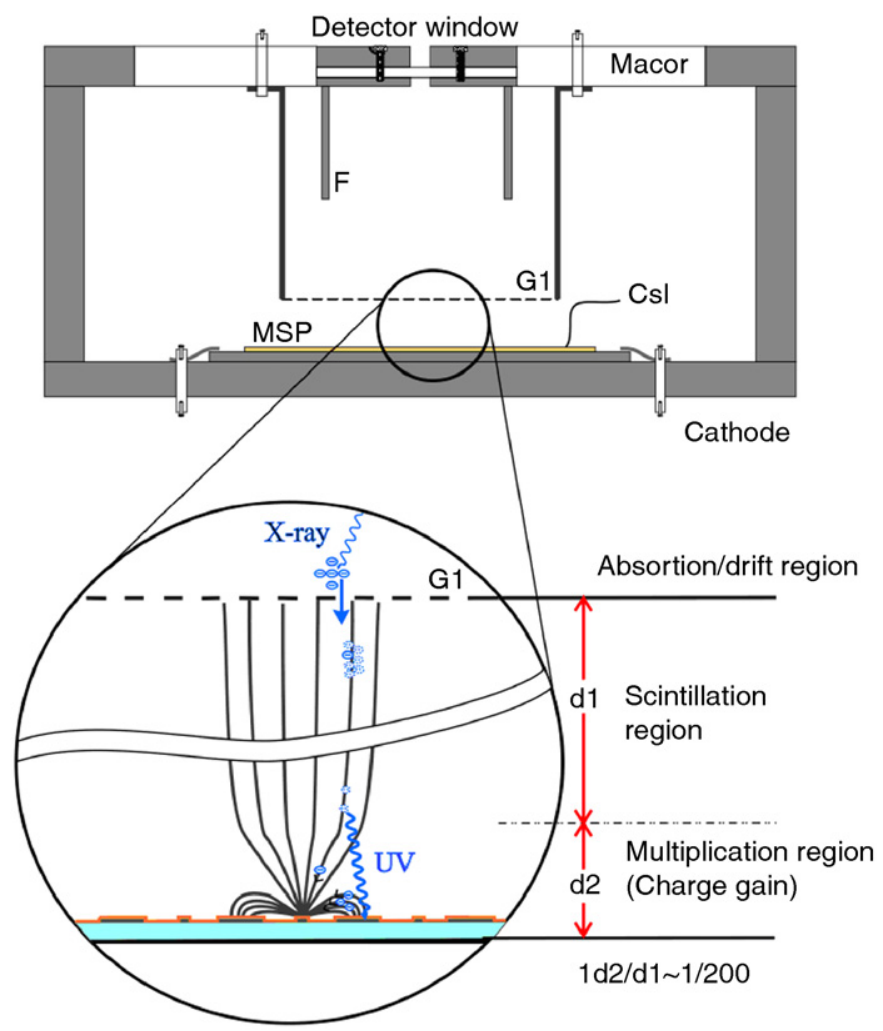

Fig. 1. Schematic of the present GPSC/MSGC hybrid detector and its operation principle. 
scintillation light as a result of the gas de-excitation processes. The scintillation light intensity is proportional to the number of primary electrons and, thus, to the X-ray energy. The VUV scintillation photons, incident on the CsI-film deposited on the MSP surface, induce the emission of photoelectrons from the active areas, the cathode strips. The photoelectrons undergo charge avalanche multiplication in the intense electric field around the anodes, being the resulting charge signal proportional to the energy of the absorbed X-ray. The CsI-coated MSP serves simultaneously as the collection electrode for the primary electron cloud, as the photosensor for the GPSC VUV scintillation and as the amplification stage for the photoelectrons. While the upper region, $d_{1}$, acts as the uniform-field scintillation region of a conventional GPSC, region $d_{2}$ $(<50 \mu \mathrm{m})$ acts as a standard MSGC. This hybrid system operates as a GPSC, rather than a MSGC [7,8].

The MSP is a CERN model MS- $4,3 \times 3 \mathrm{~cm}^{2}$ in active area, with $10-\mu \mathrm{m}$ anodes and $80-\mu \mathrm{m}$ cathodes in a $200-\mu \mathrm{m}$ pitch, fabricated with a $0.2-\mu \mathrm{m}$ chromium film deposited on a $500-\mu \mathrm{m}$ Desag D263 glass substrate. The backplane is an unstructured layer of $0.1-\mu \mathrm{m}$ chromium. A 500 -nm thick layer of high-purity CsI was vacuum evaporated onto the MSP surface. Special care was taken to prevent water contamination by heating the MSP prior to CsI evaporation, reducing its exposing time to air to less than $10 \mathrm{~min}$ and heating the CsI film under vacuum after the CsI-MSP had been placed inside the detector [12].

The detector was filled at different xenon pressures, from atmospheric pressure up to $10 \mathrm{bar}$, and the pressure was kept constant during each set of measurements. The gas inlet and outlet ports of the detector are interconnected through a U-tube, one of the arms being filled with nonevaporable getter (SAES Getters, St 707/washer/833). The getter heating was done by temperature-controlled electric heating tapes placed on the outside of the tube arm, maintaining the operating temperature steady in the range of $100-250^{\circ} \mathrm{C}$. The filling gas was continuously purified, maintained in circulation by convection through the getters, and the pressure was continuously monitored.

A 2-mm collimated $\mathrm{X}$-ray beam from a ${ }^{109} \mathrm{Cd}$ radioactive source, or a $59.6-\mathrm{keV}$ gamma-ray beam from a ${ }^{241} \mathrm{Am}$ radioactive source were used to induce detector pulses. The charge signals of the MSP anode strips were fed through a Canberra 2006 charge-to-voltage preamplifier (sensitivity of $235 \mathrm{mV} / 10^{6}$ ion pairs) and a Tennelec TC243 linear amplifier ( $8 \mu$ s peaking time constants) to a 1024-multichannel analyser. For peak amplitude and energy-resolution measurements, pulse-height distributions were fitted to a Gaussian superimposed on a linear background, from which the centroid and the full-width-at-half-maximum were determined.

\section{Experimental results and discussion}

We experienced some difficulties in obtaining a stable operation of the CsI-MSP when the CsI film covered the ends of the cathode strips which, in this MSP model, were not round. After detector operation, microdischarge paths were observed on the CsI film in these regions. Therefore, we used a $2.9-\mathrm{cm}$ diameter mask to evaporate the CsI film onto the MSP, achieving, this way, a stable detector operation. In addition, we also experienced some difficulties in obtaining efficient gas purification by gas convection through the getters, at high pressures. Getter re-activation $\left(1 \mathrm{~h}\right.$ at $\left.350^{\circ} \mathrm{C}\right)$ was performed at the beginning of each set of measurements and higher operation temperatures were used in the getters (between 200 and $250{ }^{\circ} \mathrm{C}$ ), for pressure fillings above 4 bar.

A compromise had to be established between the reduced electric field, $E / p$, i.e. the electric field divided by the gas pressure, applied to the drift region, the maximum reduced electric field that could be applied to the scintillation region and the maximum voltage that could be applied to the detector before electric breakdown occurred. Therefore, the reduced electric field used in the drift region was around $0.8 \mathrm{~V} \mathrm{~cm}^{-1} \mathrm{Torr}^{-1}$ for $1-3 \mathrm{bar}$, $0.2 \mathrm{~V} \mathrm{~cm}^{-1} \mathrm{Torr}^{-1}$ for 3 and $4 \mathrm{bar}$, and $0.1 \mathrm{~V} \mathrm{~cm}^{-1} \mathrm{Torr}^{-1}$ for 8 and 10 bar. Nevertheless, we experimentally determined that significant detector amplitudes and energy resolution degradations only occur for $E / p$ values below $\sim 0.1 \mathrm{~V} \mathrm{~cm}^{-1}$ Torr $^{-1}$. Below this value, pure xenon is very susceptible to contamination with impurities, at high pressures [17].

In Fig. 2 we depict the detector relative pulse amplitude as a function of reduced electric field, $E / p$, in the scintillation region, using the $22-\mathrm{keV}$ peak. For each pressure, the voltage difference applied between anode and cathode strips, $V_{\mathrm{a}}$, of the MSP was set to a value typically within $10 \mathrm{~V}$ from those depicted in Table 1. For Xe fillings from 1 to 4 bar, the detector amplitude was normalized to that obtained for $E / p$ below $1 \mathrm{~V} \mathrm{~cm}^{-1} \mathrm{Torr}^{-1}$ (the xenon scintillation threshold), i.e. to the gain due to

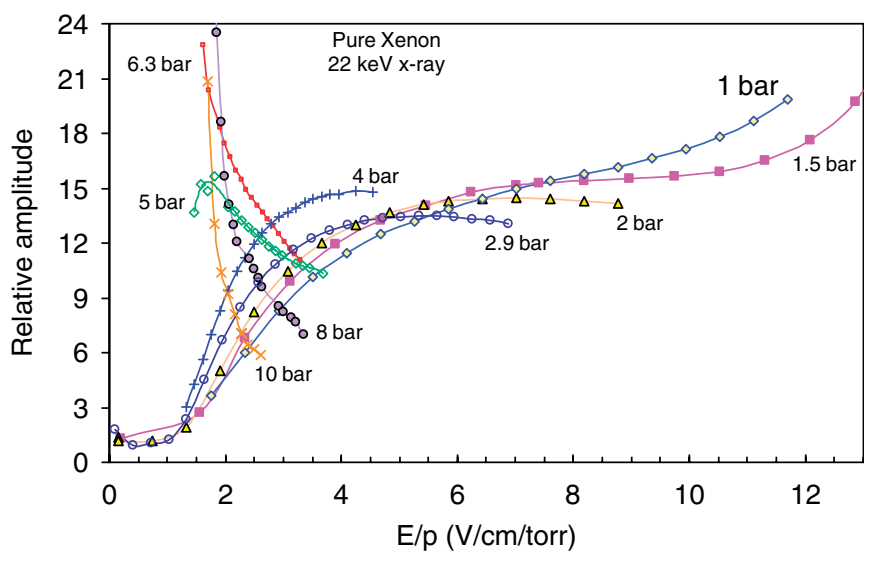

Fig. 2. Detector relative amplitude as a function of $E / p$ in the scintillation region, for the studied xenon pressures and for 22-keV X-rays. For each pressure, the $V_{\text {a }}$ value used in the MSP was set to a value within $10 \mathrm{~V}$ from those depicted in Table 1. For 1-4-bar filling pressures, the detector amplitude was normalized to that obtained for $\mathrm{E} / \mathrm{p}$ below $1 \mathrm{~V} \mathrm{~cm}^{-1} \mathrm{Torr}^{-1}$, while for the other pressure values the detector amplitude has the same normalization as for 1 bar. 
Table 1

Operating conditions for the lowest energy resolutions achieved with the present detector

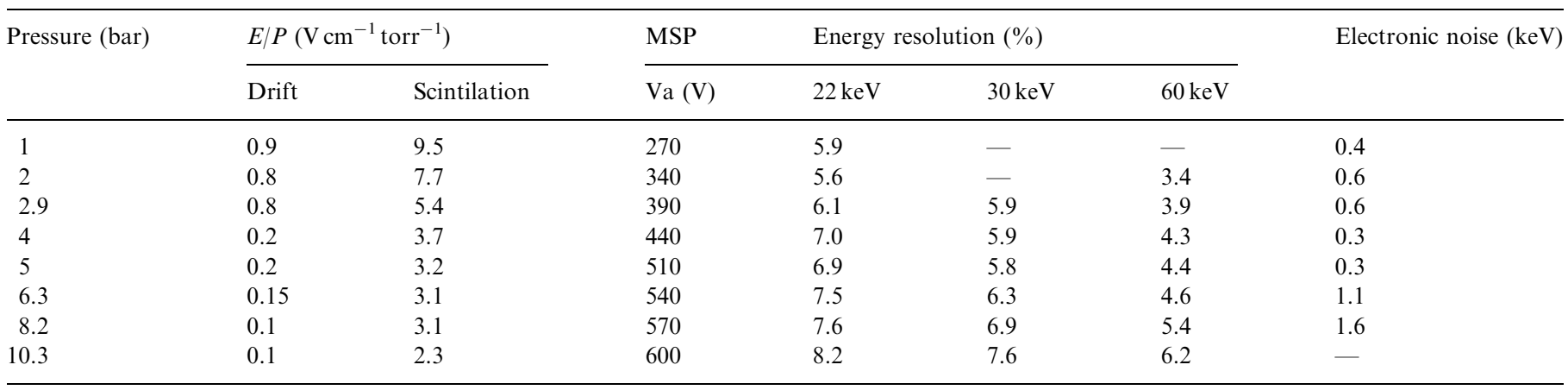

the avalanche produced only by primary electrons. This normalization is important, for it gives directly the net gain resulting from the scintillation amplification mechanisms (scintillation production plus photoelectron extraction and collection efficiency), i.e. the number of collected photoelectrons per primary electron.

This normalization was not possible for pressures above 5 bar for which, at low $E / p$ values in the scintillation region, the detector amplitude has a fast increase with decreasing $E / p$, eventually leading to instabilities and breakdown. We attribute this behavior to optical positive feedback. The scintillation produced in the avalanche around the anode strips increases with increasing pressure, since the applied $V_{\mathrm{a}}$ is higher for higher pressures. Additionally, the electric field at the photocathode surface increases with decreasing $E / p[7,18]$, resulting in a more efficient photoelectron extraction and collection [7,18]. In addition, as the $E / p$ decreases, the photoelectron paths rise higher above the photocathode plane. This results in an increase of the solid angle subtended by the cathode strips (the photocathode active area) and, thus, in an increase of the positive photon feedback [18]. Therefore, for pressures above 5 bar, the detector relative amplitudes are normalized to those of 1 bar. This behavior was confirmed in the repeated operation of this detector, with different MSPs and/or CsI-photocathodes, and a similar GPSC/MSGC hybrid detector.

As seen in Fig. 2, there is a large deviation from the linear behaviour of standard GPSCs [6] for values of $E / p$ above the scintillation threshold. This effect was already studied in Refs. [7,18]. The increase of the electric field in the scintillation region decreases the electric field at the photocathode surface, reducing the number of photoelectrons per VUV photon that escape from the photocathode. Thus, increasing the number of the produced VUV photons, by increasing $E / p$, does not result in an effective increase in the collected photoelectrons. This effect becomes a lot more significant as the pressure increases, as shown in Fig. 2. Fig. 2 also demonstrates that gains above one order of magnitude may be achieved through the scintillation process. If we take into account that, for the same $E / p$, the number of VUV photons produced in the

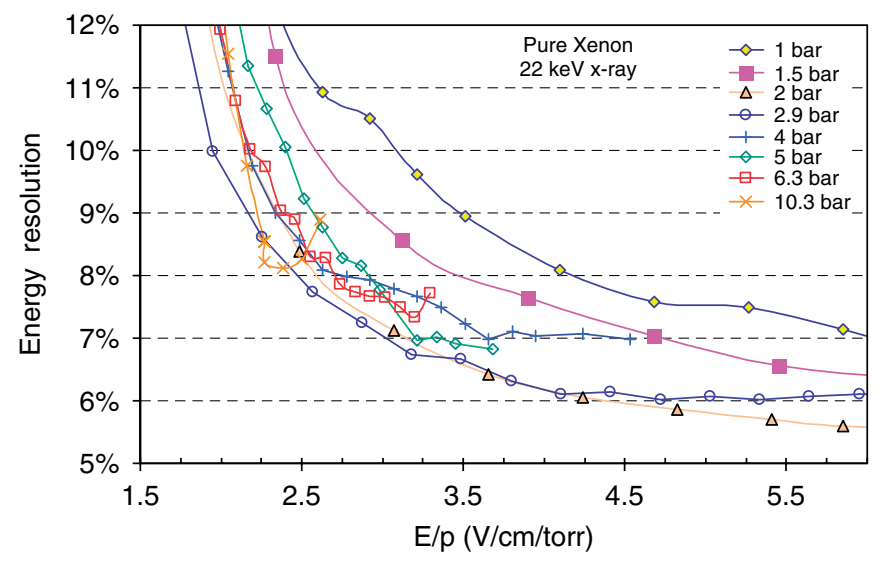

Fig. 3. Detector energy resolution for 22-keV X-rays as a function of $E / p$ in the scintillation region, for the different pressures and for the same conditions of Fig. 2.

scintillation region increases linearly with pressure, Fig. 2 shows that the number of collected photoelectrons per VUV photon decreases with pressure. This is due to photoelectron backscattering in elastic collisions with the gas atoms, as discussed in Ref. [19].

In Fig. 3, we present the detector energy resolution, FWHM, as a function of $E / p$, for $22-\mathrm{keV}$ X-rays and for the different gas-pressure fillings. For low scintillation outputs, i.e. low $E / p$, the energy resolution of GPSCs depends strongly on the number of VUV photons produced in the scintillation region, presenting a fast decrease with increasing $E / p$. As the number of VUV photons increases, this dependence becomes less significant. The fluctuations in the number of collected photoelectrons per primary electron, as well as in the photoelectron avalanche gain, become the main factors responsible for the energy resolution [6]. Since, for higher filling pressures the scintillation output is larger, its contribution to the energy resolution becomes negligible for lower $E / p$. This explains why the $E / p$ for which the lowest energy resolution is achieved decreases with the gas pressure, being around $10,8,5,4,3$ and $2.5 \mathrm{~V} \mathrm{~cm}^{-1}$ Torr $^{-1}$ for $1,2,3,4,8$ and 10 bar, respectively. This trend, of 
lowest energy resolutions for lower reduced electric fields with increasing xenon pressure, is also present in electron avalanche proportional counters [17]. For 22-kev X-rays, the lowest energy resolutions obtained with the GPSC/ MSGC hybrid detector were about $6 \%$ for 1 bar, improving to $5.5 \%$ for 2 bar, then degrading to $6 \%, 7 \%, 7.6 \%$ and $8.2 \%$ for $3,5,8$ and 10 bar, respectively. We attribute the more significant degradation of this resolution, at high pressures, to the decrease in the number of detected photoelectrons per VUV photons and to the lower purification efficiency of xenon in the getters. Thus, the resolution obtained for those pressures should be considered as upper limits.

In Fig. 4, the detector relative amplitude and energy resolution is depicted as a function of $V_{\mathrm{a}}$ for the different pressures and for the $E / p$ values listed in Table 1. The maximum $V_{\text {a }}$ voltages that can be applied, at the discharge limit, were not reached. As observed in Fig. 4, the $V_{\text {a }}$ values delivering the lowest energy resolutions are limited by the onset of the additional fluctuations introduced by the optical feedback resulting from the additional VUV photons produced in the electron avalanches around the anode strips, which degrade the energy resolution. This allows the maximum amplitude achieved for the lowest energy resolution to be rather independent of the gas pressure, in opposition to gas avalanche devices, where the maximum applied voltages are much higher and only limited by sparking, resulting in a large gain dependence on pressure [3-5]. This feedback effect depends on the total number of photons produced in the avalanche, which increase with pressure, via $V_{\mathrm{a}}$, and on the number of photoelectrons, per VUV photon, that are transmitted to the anode strips, which decrease with increasing pressure.

In Fig. 5, the detector amplitude, normalized to that of 1 bar, is depicted as a function of pressure for the conditions of $E / p$ and $V_{\mathrm{a}}$ that deliver the lowest energy resolutions for each gas pressure (Table 1). We can observe that after an initial fast decrease of about $40 \%$ when the pressure increases from 1 to 2 bar, the amplitude decreases

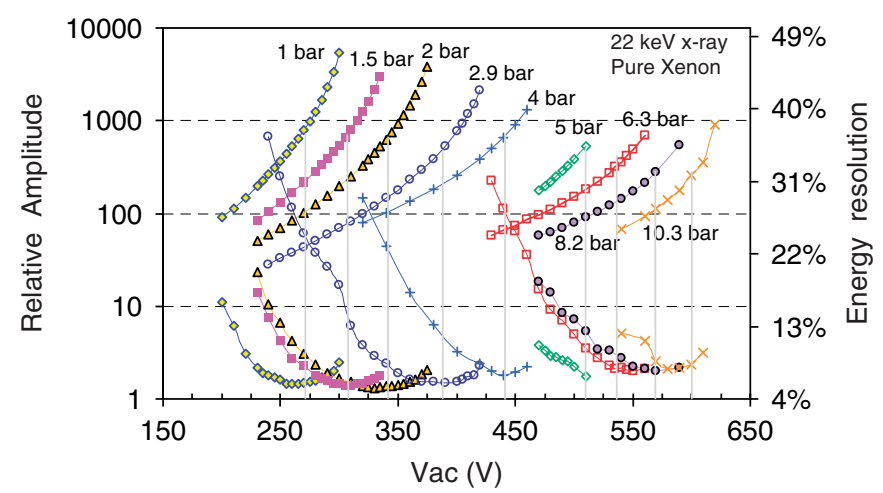

Fig. 4. Detector relative amplitude and energy resolution for $22-\mathrm{keV} X-$ rays as a function of $V_{\mathrm{a}}$ for the studied xenon pressures and for the $E / p$ values listed in Table 1 .

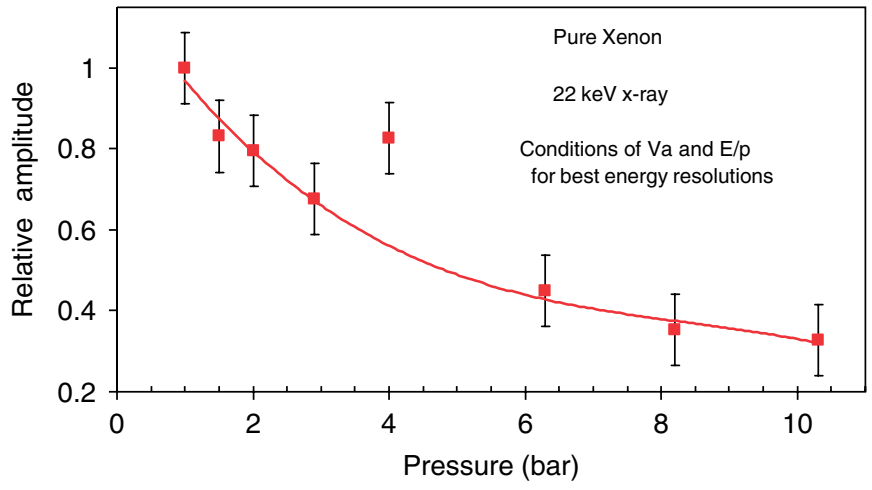

Fig. 5. Detector relative amplitude, normalized to that of $1 \mathrm{bar}$, as a function of xenon pressure, for the $E / p$ and $V_{\mathrm{a}}$ values listed in Table 1.
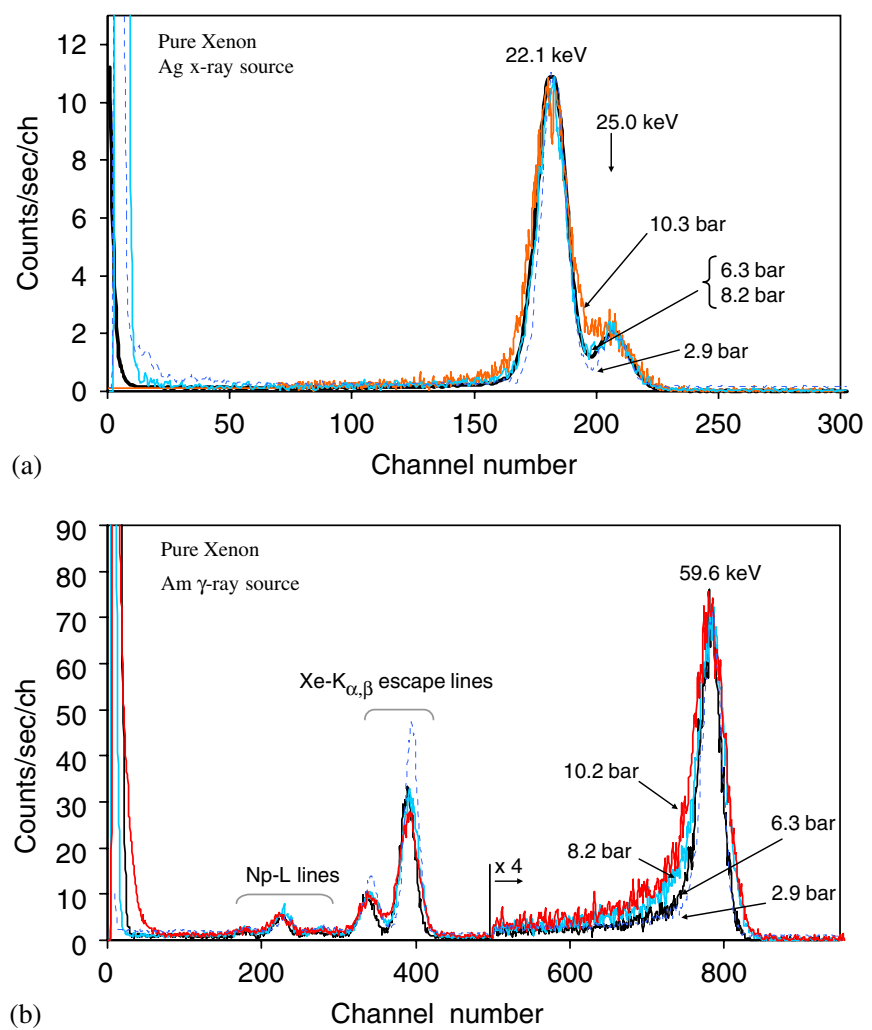

Fig. 6. Typical pulse-height distributions obtained with the detector for the X-rays and $\gamma$-rays from ${ }^{109} \mathrm{Cd}$ and ${ }^{241} \mathrm{Am}$ radioactive sources, for different xenon filling pressures.

slowly with increasing pressure, being reduced by about $60 \%$ and $70 \%$ for pressures of 8 and 10 bar, respectively.

Fig. 6 depicts the typical detector pulse-height distributions obtained for ${ }^{109} \mathrm{Cd}(a)$ and ${ }^{241} \mathrm{Am}(b)$ radioactive sources for different gas pressures. Low plateau levels with good energy resolutions show the good potential of the xenon-GPSC/MSGC hybrid detector operating at high pressures. For $60 \mathrm{keV}$, in particular, the increase in the detection efficiency $(70 \%$ at 10 bar) for the events due to full-energy absorption, results in a better peak-to-plateau ratio and in less intense escape peaks. The detector energy 
resolutions, FWHM, for $60-\mathrm{keV}$ photons degrades from $3.4 \%$ at 2 bar to $3.9 \%, 4.6 \%, 5.4 \%$ and $6.2 \%$ at $3,6,8$ and 10 bar, respectively. The detector-energy resolution degradation at high pressures can be reduced by improving the gas purification efficiency and the electrical insulation, to allow the use of higher reduced electric fields in the drift region.

Table 1 summarizes the operating conditions and performance for which the lowest energy resolutions are achieved, with the present detector. The obtained energy resolutions are better than those of electron avalanche detectors. At filling pressures of 1,5 and 10-bar, typical values obtained with PCs [17] are $7.7 \%, 8.3 \%$ and $9.6 \%$, and $5.5 \%, 6.0 \%$ and $6.5 \%$ for 22 and $60 \mathrm{keV}$ photons, respectively. For an MSGC with 2 bar Xe-filling [20], energy resolutions of $9 \%$ and $6 \%$ were obtained for 22and $60-\mathrm{keV}$ photons, while for a GEM + MSGC detector filled with 4-bar Xe [21], an energy resolution of $8 \%$ was obtained for $60-\mathrm{keV}$ photons. For standard Xe-GPSCs operated with PMTs, typical energy resolutions of $6.1 \%$ and $3.6 \%$ were achieved for $30-$ and $60-\mathrm{keV}$ photons, respectively, at $5.5 \mathrm{bar}$, while at 9 bar the resolution degrades to $7.6 \%$ and $3.6 \%$, respectively $[22,23]$.

\section{Conclusions}

We investigated the performance characteristics of the xenon-GPSC/MSGC hybrid detector when operated at high pressures, ranging from 1 to $10 \mathrm{bar}$, for the detection of $22-$ and $60-\mathrm{keV}$ photons. High pressure gas fillings extend the detection range to hard X-rays at the cost of small detector performance degradation. The lowest energy resolutions were achieved for 2 bar, but competitive energy resolutions were still achieved in the 4- to 6-bar range. Compared to detectors based only on electron avalanche amplification, the hybrid detector presents higher gains and improved energy resolutions over all the studied pressure range. It also features safer operation conditions and a non-aging gas, easy to handle and to purify in sealed detectors. For the optimum operating conditions, the detector total gain decreases by only $70 \%$ when the pressure increases from 1 to 10 bar.

The optimal detector operation and the applied $V_{\mathrm{a}}$ are limited by optical photon feedback, since the photocathode is not concealed from the photosensor electron-avalanche region. On the other hand, the use of $V_{\text {a }}$ values well below the MSP discharge limit results in increased stability and reduced ageing. The pressure increase allows the increase of $V_{\mathrm{a}}$, increasing the amount of feedback scintillation but, in opposition, reduces the number of photoelectrons per VUV photon that are transmitted to the anode strips. The combination of these effects result in a small pressure dependence of the maximum amplitude achieved for the lowest energy resolution. In spite of the fact that the scintillation produced in the GPSC increases with pressure, the $E / p$ values delivering the lowest energy resolutions decrease with pressure, since the electric field intensity in the scintillation region also affects the electric field on the cathode surface and the photoelectron collection efficiency.

Decoupling the electric field of the GPSC scintillation region from that of the MSGC drift region will provide conditions to implement more efficient fields above the MSP cathodes, which will improve photoelectron collection efficiency. In principle, this could be achieved by simply using an extra grounded grid, placed a few millimetres above the MSP. However, we could not reach a stable operation in such detector, up to now.

\section{Acknowledgments}

This work was carried out in the Instrumentation Centre of the Physics Department of the University of Coimbra and it was supported by Project POCTI/FP/FNU/50212/03 through FEDER and FCT (Lisbon) programs. We acknowledge the reviewer for the helpful comments and suggestions.

\section{References}

[1] A. Oed, Nucl. Instr. and Meth. A 263 (1988) 351.

[2] F. Sauli, Nucl. Instr. and Meth. A 386 (1997) 531.

[3] J. Nickles, H. Bräuning, A. Bräuning-Demian, V. Dangendorf, K. Rauschnabel, H. Schmidt-Böcking, IEEE Trans. Nucl. Sci. NS-49 (2002) 808.

[4] A. Bondar, A. Buzulutskov, F. Sauli, L. Sheckhtman, Nucl. Instr. and Meth. A 419 (1998) 418.

[5] A. Bondar, A. Buzulutskov, L. Schekhtmann, Nucl. Instr. and Meth. A 481 (2002) 200.

[6] J.M.F. dos Santos, J.A.M. Lopes, J.F.C.A. Veloso, P.C.P.S. Simões, T.H.V.T. Dias, F.P. Santos, P.J.B.M. Rachinhas, L.F.R. Ferreira, C.A.N. Conde, X-ray Spectrom 30 (2001) 373.

[7] J.F.C.A. Veloso, J.M.F. dos Santos, C.A.N. Conde, Nucl. Instr. and Meth. A 457 (2001) 253.

[8] D.S.A.P. Freitas, J.F.C.A. Veloso, C.M.B. Monteiro, J.M.F. dos Santos, C.A.N. Conde, Nucl. Instr. and Meth. A 505 (2003) 228.

[9] A. Breskin, Nucl. Instr. and Meth. A 371 (1996) 116.

[10] K. Zeitelhack, J. Friese, R. Gernhäuser, P. Kienle, H.-J. Körner, P. Maier-Komor, S. Winkler, Nucl. Instr. and Meth. A 351 (1994) 585.

[11] K. Zeitelhack, A. Stolz, B. Bauer, C. Erbe, J. Friese, R. Gernhäuser, A. Kastenmüller, P. Kienle, H.-J. Körner, P. Maier-Komor, M. Münch, S. Winkler, Nucl. Instr. and Meth. A 371 (1996) 57.

[12] J. Va'vra, A. Breskin, A. Buzulutskov, R. Chechik, E. Shefer, Nucl. Instr. and Meth. A 387 (1997) 154.

[13] R. Bouclier, M. Capeans, G. Million, L. Ropelewski, F. Sauli, T. Temmel, G. Della Mea, G. Maggioni, V. Rigato, Nucl. Instr. and Meth A 369 (1996) 328.

[14] D. Taqqu, et al., Hyperfine Interactions 119 (1999) 311.

[15] J.F.C.A. Veloso, J.A.M. Lopes, C.A.N. Conde, L.M.P. Fernandes, E.D.C. Freitas, O. Huot, P. Knowles, F. Kottmann, F. Mulhauser, J.M.F. dos Santos, D. Taqqu, IEEE Trans. Nucl. Sci. NS-49 (2002) 899.

[16] D.S. Covita, J.F.C.A. Veloso, F.D. Amaro, J.M.F. dos Santos, C.A.N. Conde, IEEE Trans. Nucl. Sci. NS-50 (2003) 855.

[17] H. Sakurai, B.D. Ramsey, M.C. Weisskopf, Nucl. Instr. and Meth. A 307 (1991) 504.

[18] D.S.A.P. Freitas, J.F.C.A. Veloso, J.M.F. Dos Santos, C.A.N. Conde, IEEE Trans. Nucl. Sci. NS-48 (2001) 408.

[19] P.J.B.M. Rachinhas, T.H.V.T. Dias, J.A.M. Lopes, F.P. Santos, L.M.N. Távora, C.A.N. Conde, A.D. Stauffer, Dependence of the photoelectron collection efficiency in noble gases on the incident 
VUV photon energy, In: 2002-IEEE Nuclear Science Symposium Conference Records, Norfolk, VA, November. 2002.

[20] B.D. Ramsey, J.A. Apple, R.A. Austin, K.L. Dietz, T. Minamitani, J.J. Kolodziejczak, M.C. Weisskopf, Nucl. Instr. and Meth. A 383 (1996) 424.

[21] M. Li, M.S. Dixit, P.C. Johns, Nucl. Instr. and Meth. A 471 (2001) 215.
[22] A. Bolozdynya, V. Egorov, A. Koutchenkov, G. Safronov, G. Smirnov, S. Medved, V. Morgunov, Nucl. Instr. and Meth. A 385 (1997) 225.

[23] A. Bolozdynya, V. Egorov, A. Koutchenkov, G. Safronov, G. Smimov, S. Medved, V. Morgunov, IEEE Trans. Nucl. Sci. NS-44 (1997) 1046 references therein. 